Russell A. Gordon, Department of Mathematics, Whitman College, Walla

Walla, WA 99362, e-mail:gordon@whitman.edu

\title{
SOME COMMENTS ON THE MCSHANE AND HENSTOCK INTEGRALS
}

\begin{abstract}
The basic distinction between the Henstock and McShane integrals involves the location of the tags. Another way to look at this difference is through the type of set associated with each tag; intervals for the Henstock integral and finite unions of intervals for the McShane integral. Does this distinction make any difference in the definition of the Riemann integral? What happens if the intervals are replaced with arbitrary measurable sets? These questions are answered in this paper. A relationship between the Henstock integral and outer Lebesgue measure is also included.
\end{abstract}

The Henstock integral and the McShane integral have similar definitions that are based upon allowing the mesh size in the Riemann integral definition to vary from point to point rather than to be constant. This rather subtle change from the Riemann definition has a great impact on what functions are integrable. The difference between the Henstock and McShane definitions involves the location of the tags. For the Henstock integral, the tag of an interval must belong to that interval whereas for the McShane integral the tag of an interval need not belong to the interval. Consequently, it is more difficult for a function to be McShane integrable and, as it turns out, the McShane definition kicks out those functions that are not absolutely integrable. It can be shown that the McShane integral is equivalent to the Lebesgue integral and that the Henstock integral is equivalent to the restricted Denjoy integral. The fact that such powerful integrals can be defined almost as simply as the Riemann integral is of pedagogical interest to some. The main focus in this paper will be the distinction between the two definitions.

The first question that will be examined is whether or not tags in versus tags out makes any difference when using a constant mesh size. Since the

Key Words: Henstock integral, McShane integral

Mathematical Reviews subject classification: 26A39

Received by the editors September 6, 1997 
Henstock and McShane integrals are equivalent for the class of bounded functions (both are equivalent to the Lebesgue integral in this case) and a constant mesh size yields the Riemann integral, it is to be expected that the location of the tags will make no difference when the mesh is constant. However, a proof of this fact does not immediately manifest itself. We begin with some notation.

A partition $\left\{J_{k}: 1 \leq k \leq q\right\}$ of the interval $[a, b]$ is a finite collection of non-overlapping closed intervals such that $[a, b]=\bigcup_{k=1}^{q} J_{k}$. We will always assume that the intervals in a partition are listed in increasing order with respect to the number line. A tagged interval, denoted $(x,[c, d])$, consists of an interval $[c, d]$ and a tag $x \in[c, d]$. A free tagged interval is a tagged interval in which the tag is free of the restriction that it belong to the interval. For example, $(1,[1,2])$ is a tagged interval and $(0,[1,2])$ is a free tagged interval. Every tagged interval is a free tagged interval but not every free tagged interval is a tagged interval. A tagged partition (free tagged partition) of $[a, b]$ is a partition of $[a, b]$ in which each interval is a tagged (free tagged) interval. Given a positive number $\delta$, a tagged partition (free tagged partition) is said to be $\delta$-fine if each tagged (free tagged) interval $(x,[c, d])$ satisfies $[c, d] \subseteq(x-\delta, x+\delta)$. For a function $f:[a, b] \rightarrow R$ and a tagged partition (free tagged partition) $\left\{\left(x_{k}, J_{k}\right): 1 \leq k \leq q\right\}$ of $[a, b]$, the sum

$$
\sum_{k=1}^{q} f\left(x_{k}\right) \ell\left(J_{k}\right)
$$

$\left(\ell\left(J_{k}\right)\right.$ is the length of the interval $\left.J_{k}\right)$ represents the Riemann sum of $f$ over the given tagged partition (free tagged partition).

It should be clear that a function $f:[a, b] \rightarrow R$ is Riemann integrable on $[a, b]$ if and only if there exists a number $L$ with the following property: for each $\epsilon>0$ there exists a positive number $\delta$ such that

$$
\left|\sum_{k=1}^{q} f\left(x_{k}\right) \ell\left(J_{k}\right)-L\right|<\epsilon
$$

whenever $\left\{\left(x_{k}, J_{k}\right): 1 \leq k \leq q\right\}$ is a $\delta$-fine tagged partition of $[a, b]$. We would like to prove that this result remains valid when $\delta$-fine tagged partitions of $[a, b]$ are replaced with $\delta$-fine free tagged partition of $[a, b]$. In other words, it makes no difference whether or not the tags belong to the interval as long as all of the tagged intervals are $\delta$-fine. To prove this result, we need some more notation and a lemma. 
Let $f$ be a function defined on the interval $[a, b]$. Given a partition $\left\{J_{k}\right.$ : $1 \leq k \leq q\}$ of $[a, b]$, let $J_{0}=J_{1}$ and $J_{q+1}=J_{q}$ and define

$$
\begin{aligned}
m\left(f, J_{k}\right) & =\inf \left\{f(x): x \in J_{k}\right\} ; \\
m^{\&}\left(f, J_{k}\right) & =\inf \left\{f(x): x \in J_{k-1} \cup J_{k} \cup J_{k+1}\right\} ; \\
M\left(f, J_{k}\right) & =\sup \left\{f(x): x \in J_{k}\right\} ; \\
M^{\&}\left(f, J_{k}\right) & =\sup \left\{f(x): x \in J_{k-1} \cup J_{k} \cup J_{k+1}\right\} ;
\end{aligned}
$$

for $1 \leq k \leq q$. The norm of the partition $\left\{J_{k}: 1 \leq k \leq q\right\}$ is the maximum of the lengths of the intervals in the partition. Recall that a function $f:[a, b] \rightarrow R$ is Riemann integrable on $[a, b]$ if and only if there exists $\delta>0$ such that

$$
\sum_{k=1}^{q}\left(M\left(f, J_{k}\right)-m\left(f, J_{k}\right)\right) \ell\left(J_{k}\right)<\epsilon
$$

whenever $\left\{J_{k}: 1 \leq k \leq q\right\}$ is a partition of $[a, b]$ with norm less than $\delta$. This is one version of the Cauchy criterion for Riemann integrability.

Lemma 1. If $f:[a, b] \rightarrow R$ is Riemann integrable on $[a, b]$, then for each $\epsilon>0$ there exists $\eta>0$ such that

$$
\sum_{k=1}^{q}\left(M^{\&}\left(f, J_{k}\right)-m^{\&}\left(f, J_{k}\right)\right) \ell\left(J_{k}\right)<\epsilon
$$

for every partition $\left\{J_{k}: 1 \leq k \leq q\right\}$ of $[a, b]$ with norm less than $\eta$ and

$$
\sum_{k=1}^{q} m^{\&}\left(f, J_{k}\right) \ell\left(J_{k}\right) \leq \int_{a}^{b} f \leq \sum_{k=1}^{q} M^{\&}\left(f, J_{k}\right) \ell\left(J_{k}\right)
$$

for each such partition of $[a, b]$.

Proof. Let $\epsilon>0$. Since $f$ is Riemann integrable on $[a, b]$, there exists $\delta>0$ such that

$$
\sum_{j=1}^{n}\left(M\left(f, I_{j}\right)-m\left(f, I_{j}\right)\right) \ell\left(I_{j}\right)<\epsilon / 3
$$

whenever $\left\{I_{j}: 1 \leq j \leq n\right\}$ is a partition of $[a, b]$ with norm less than $\delta$. Let $\eta=\delta / 3$ and suppose that $\left\{J_{k}: 1 \leq k \leq q\right\}$ is a partition of $[a, b]$ with norm less than $\eta$. Let $I_{k}=J_{k-1} \cup J_{k} \cup J_{k+1}$ for $1 \leq k \leq q$ and note that

$$
m^{\&}\left(f, J_{k}\right)=m\left(f, I_{k}\right) \quad \text { and } \quad M^{\&}\left(f, J_{k}\right)=M\left(f, I_{k}\right)
$$


for $1 \leq k \leq q$. Define sets

$$
\begin{aligned}
& S_{0}=\{k: 1 \leq k \leq q \text { and } k \equiv 0 \quad(\bmod 3)\} \\
& S_{1}=\{k: 1 \leq k \leq q \text { and } k \equiv 1 \quad(\bmod 3)\} ; \\
& S_{2}=\{k: 1 \leq k \leq q \text { and } k \equiv 2 \quad(\bmod 3)\}
\end{aligned}
$$

Now $\left\{I_{k}: k \in S_{0}\right\}$ is a finite collection of non-overlapping intervals in $[a, b]$ with norm less than $\delta$. Although this collection may not be a partition of $[a, b]$, it still follows that (since adding more terms only increases the sum)

$$
\sum_{k \in S_{0}}\left(M^{\&}\left(f, J_{k}\right)-m^{\&}\left(f, J_{k}\right)\right) \ell\left(J_{k}\right) \leq \sum_{k \in S_{0}}\left(M\left(f, I_{k}\right)-m\left(f, I_{k}\right)\right) \ell\left(I_{k}\right)<\epsilon / 3 .
$$

The same inequality is valid for sums with $k \in S_{1}$ and $k \in S_{2}$. Therefore,

$$
\begin{aligned}
\sum_{k=1}^{q}\left(M^{\&}\left(f, J_{k}\right)-m^{\&}\left(f, J_{k}\right)\right) \ell\left(J_{k}\right) & =\sum_{k \in S_{0}} "+\sum_{k \in S_{1}} "+\sum_{k \in S_{2}} " \\
& <\epsilon / 3+\epsilon / 3+\epsilon / 3=\epsilon .
\end{aligned}
$$

The second part of the conclusion follows from the inequality

$$
\begin{aligned}
\sum_{k=1}^{q} m^{\&}\left(f, J_{k}\right) \ell\left(J_{k}\right) & \leq \sum_{k=1}^{q} m\left(f, J_{k}\right) \ell\left(J_{k}\right) \leq \int_{a}^{b} f \\
& \leq \sum_{k=1}^{q} M\left(f, J_{k}\right) \ell\left(J_{k}\right) \leq \sum_{k=1}^{q} M^{\&}\left(f, J_{k}\right) \ell\left(J_{k}\right) .
\end{aligned}
$$

This completes the proof.

Theorem 2. A function $f:[a, b] \rightarrow R$ is Riemann integrable on $[a, b]$ if and only if there exists a number $L$ with the following property: for each $\epsilon>0$ there exists a positive number $\delta$ such that

$$
\left|\sum_{k=1}^{q} f\left(x_{k}\right) \ell\left(J_{k}\right)-L\right|<\epsilon
$$

whenever $\left\{\left(x_{k}, J_{k}\right): 1 \leq k \leq q\right\}$ is a $\delta$-fine free tagged partition of $[a, b]$.

Proof. Suppose that $f$ is Riemann integrable on $[a, b]$ and let $\epsilon>0$. Choose $\eta>0$ according to the previous lemma and let $\delta=\eta / 4$. Suppose that $\left\{\left(x_{i},\left[c_{i-1}, c_{i}\right]\right): 1 \leq i \leq n\right\}$ is a $\delta$-fine free tagged partition of $[a, b]$. Since 
the length of each interval $\left[c_{i-1}, c_{i}\right]$ is less than $\eta / 2$, there exists an increasing set $\left\{i_{k}: 0 \leq k \leq q\right\}$ of indices such that

$$
0=i_{0}<i_{1}<i_{2}<\cdots<i_{q}=n \text { and } \eta / 2<c_{i_{k}}-c_{i_{k-1}}<\eta
$$

for $1 \leq k \leq q$. Let $J_{k}=\left[c_{i_{k-1}}, c_{i_{k}}\right]$ for $1 \leq k \leq q$ and note that $\left\{J_{k}: 1 \leq\right.$ $k \leq q\}$ is a partition of $[a, b]$ with norm less than $\eta$. Fix an integer $k$ such that $1 \leq k \leq q$. Note that $\left\{\left[c_{i-1}, c_{i}\right]: i_{k-1}<i \leq i_{k}\right\}$ is a partition of $J_{k}$. In addition, since $\delta=\eta / 4$ and $\ell\left(J_{k}\right)>\eta / 2$, we find that $x_{i} \in J_{k-1} \cup J_{k} \cup J_{k+1}$ for each $i_{k-1}<i \leq i_{k}$. For these values of $i$,

$$
m^{\&}\left(f, J_{k}\right)\left(c_{i}-c_{i-1}\right) \leq f\left(x_{i}\right)\left(c_{i}-c_{i-1}\right) \leq M^{\&}\left(f, J_{k}\right)\left(c_{i}-c_{i-1}\right),
$$

and it follows that

$$
m^{\&}\left(f, J_{k}\right) \ell\left(J_{k}\right) \leq \sum_{i=i_{k-1}+1}^{i_{k}} f\left(x_{i}\right)\left(c_{i}-c_{i-1}\right) \leq M^{\&}\left(f, J_{k}\right) \ell\left(J_{k}\right) .
$$

Summing these inequalities for $1 \leq k \leq q$ yields

$$
\sum_{k=1}^{q} m^{\&}\left(f, J_{k}\right) \ell\left(J_{k}\right) \leq \sum_{i=1}^{n} f\left(x_{i}\right)\left(c_{i}-c_{i-1}\right) \leq \sum_{k=1}^{q} M^{\&}\left(f, J_{k}\right) \ell\left(J_{k}\right) .
$$

Using both parts of the conclusion of the previous lemma, we find that

$$
\left|\sum_{i=1}^{n} f\left(x_{i}\right)\left(c_{i}-c_{i-1}\right)-\int_{a}^{b} f\right|<\epsilon
$$

where $\int_{a}^{b} f$ is the Riemann integral of $f$. This completes the proof.

Theorem 2 shows that for a constant $\delta$ the location of the tags does not affect the integral. With a variable $\delta$, this result is no longer valid. If the tags are required to be in the interval, the Henstock integral is obtained; if the tags are allowed to move out of the interval, the McShane integral is obtained. The practical distinction that then occurs is that in the McShane case a given tag may be associated with many intervals and these intervals can, to a certain extent, be scattered about. It is this property that prohibits nonabsolutely integrable functions from being McShane integrable (see Chapter 10 of [1]). There is another way to view this difference between the two integrals. Rather than focusing on intervals and whether or not the tag belongs to an interval, we can consider the set that is associated with a tag. In the Henstock definition the set associated with a given tag is a closed interval whereas in the McShane 
definition the set associated with a given tag is a figure, a finite union of nonoverlapping closed intervals. Hence, the Henstock definition involves tagged intervals and the McShane definition involves tagged figures. We will now formalize this distinction.

A degenerate interval is an interval consisting of a single point; the point $c$ can be written in interval form as $[c, c]$. In the sequel, we will include degenerate intervals in the collection of closed intervals. For the record, the length of a degenerate interval is zero. A figure is a finite union of nonoverlapping closed intervals and the length of a figure is the sum of the lengths of the intervals that make up the figure. Two figures are said to be nonoverlapping if their intersection is a finite set. A tagged figure, denoted $(x, V)$, consists of a figure $V$ and a point $x \in V$. A figure partition of $[a, b]$ is a finite collection of non-overlapping figures whose union is $[a, b]$. A tagged figure partition of $[a, b]$ is a figure partition of $[a, b]$ in which each figure is a tagged figure. For the sake of illustration, a typical tagged figure might look like $(0, V)$ where

$$
V=[0,0] \cup\left[\frac{1}{3}, \frac{1}{2}\right] \cup\left[\frac{1}{5}, \frac{1}{4}\right] \cup\left[\frac{1}{7}, \frac{1}{6}\right] \cup \cdots \cup\left[\frac{1}{101}, \frac{1}{100}\right] .
$$

Given a positive function $\delta$ defined on $[a, b]$ (which of course may be constant), a tagged figure partition is said to be $\delta$-fine if each tagged figure $(x, V)$ satisfies $V \subseteq(x-\delta(x), x+\delta(x))$. For a function $f:[a, b] \rightarrow R$ and a tagged figure partition $\left\{\left(x_{k}, V_{k}\right): 1 \leq k \leq q\right\}$ of $[a, b]$, the sum

$$
\sum_{k=1}^{q} f\left(x_{k}\right) \ell\left(V_{k}\right)
$$

represents the Riemann sum of $f$ over the given tagged figure partition.

Using this notation and terminology, we can rephrase the definitions of the Riemann, Henstock, and McShane integrals. These statements must be read carefully as only one or two words (number or function, interval or figure) make all the difference. Definition A is a rephrasing of Theorem 2.

Definition A. A function $f:[a, b] \rightarrow R$ is Riemann integrable on $[a, b]$ if and only if there exists a number $L$ with the following property: for each $\epsilon>0$ there exists a positive number $\delta$ such that

$$
\left|\sum_{k=1}^{q} f\left(x_{k}\right) \ell\left(V_{k}\right)-L\right|<\epsilon
$$

whenever $\left\{\left(x_{k}, V_{k}\right): 1 \leq k \leq q\right\}$ is a $\delta$-fine tagged figure partition of $[a, b]$. 
Definition B. A function $f:[a, b] \rightarrow R$ is Henstock integrable on $[a, b]$ if and only if there exists a number $L$ with the following property: for each $\epsilon>0$ there exists a positive function $\delta$ defined on $[a, b]$ such that

$$
\left|\sum_{k=1}^{q} f\left(x_{k}\right) \ell\left(I_{k}\right)-L\right|<\epsilon
$$

whenever $\left\{\left(x_{k}, I_{k}\right): 1 \leq k \leq q\right\}$ is a $\delta$-fine tagged (interval) partition of $[a, b]$.

Definition C. A function $f:[a, b] \rightarrow R$ is McShane integrable on $[a, b]$ if and only if there exists a number $L$ with the following property: for each $\epsilon>0$ there exists a positive function $\delta$ defined on $[a, b]$ such that

$$
\left|\sum_{k=1}^{q} f\left(x_{k}\right) \ell\left(V_{k}\right)-L\right|<\epsilon
$$

whenever $\left\{\left(x_{k}, V_{k}\right): 1 \leq k \leq q\right\}$ is a $\delta$-fine tagged figure partition of $[a, b]$.

It is often stated, quite correctly, that both the Henstock and McShane integrals are generalizations of the Riemann integral. Both generalize the Riemann integral by considering a positive function rather than a positive number. It is evident that Definition B for the Henstock integral is almost identical to the definition of the Riemann integral. For the McShane integral, it is also necessary to allow the tags to come out of their intervals. The advantage of Definition A (which is Theorem 2) is that it keeps the focus on the positive function - there is no need to consider misplaced tags. Thus, Definition $\mathrm{C}$ for the McShane integral is almost identical to an equivalent definition for the Riemann integral.

In the case of a constant $\delta$, there is no difference between tagged interval partitions and tagged figure partitions as far as the collection of integrable functions is concerned while in the case of a variable $\delta$, the difference between tagged interval partitions and tagged figure partitions is quite dramatic. What happens if we allow other sets to form the partition? In particular, suppose that we use measurable sets rather than figures. Does this change the collection of integrable functions? At this stage in the paper, the reader should have no trouble giving a definition for a $\delta$-fine tagged measurable partition of $[a, b]$. (For consistency, we will still say that two measurable sets are nonoverlapping if their intersection is a finite set.) As the next two theorems indicate, the transition to measurable sets makes no difference. We will use $\mu(E)$ to represent the Lebesgue measure of the set $E$.

Theorem 3. A function $f:[a, b] \rightarrow R$ is Riemann integrable on $[a, b]$ if and only if there is a number $L$ with the following property: for each $\epsilon>0$ there 
exists a positive number $\delta$ such that

$$
\left|\sum_{i=1}^{n} f\left(x_{i}\right) \mu\left(E_{i}\right)-L\right|<\epsilon
$$

whenever $\left\{\left(x_{i}, E_{i}\right): 1 \leq i \leq n\right\}$ is a $\delta$-fine tagged measurable partition of $[a, b]$.

Proof. Suppose that $f$ is Riemann integrable on $[a, b]$ and let $\epsilon>0$. Choose $\eta>0$ according to Lemma 1 and let $\delta=\min \{\eta / 2,(b-a) / 3\}$. Since

$$
\frac{b-a}{\delta}-\frac{b-a}{2 \delta}=\frac{b-a}{2 \delta} \geq \frac{3}{2}
$$

there exists a positive integer $q$ such that

$$
\frac{b-a}{2 \delta}<q<\frac{b-a}{\delta} \quad \text { or } \quad \delta<\frac{b-a}{q}<2 \delta .
$$

Let $c_{k}=a+k(b-a) / q$ for $0 \leq k \leq q$ and let $J_{k}=\left[c_{k-1}, c_{k}\right]$ for $1 \leq k \leq q$. Note that the collection $\left\{J_{k}: 1 \leq k \leq q\right\}$ is a partition of $[a, b]$ with norm less than $\eta$. Suppose that $\left\{\left(x_{i}, E_{i}\right): 1 \leq i \leq n\right\}$ is a $\delta$-fine tagged measurable partition of $[a, b]$. Without loss of generality, we may assume that for each $i$ there is an index $k$ such that $E_{i} \backslash\left\{x_{i}\right\} \subseteq J_{k}$. To see this, simply replace $\left(x_{i}, E_{i}\right)$ with the tagged measurable sets from the collection $\left\{\left(x_{i},\left(E_{i} \cap J_{k}\right) \cup\left\{x_{i}\right\}\right): 1 \leq k \leq q\right\}$. (Except for the trivial $\left(x_{i},\left\{x_{i}\right\}\right)$, there are at most three sets in this collection; $E_{i}$ has nonempty intersection with at most three of the intervals $J_{k}$.) Finally, let $S_{k}=\left\{i: E_{i} \backslash\left\{x_{i}\right\} \subseteq J_{k}\right\}$ for $1 \leq k \leq q$. Note that the symmetric difference

$$
\left(\bigcup_{i \in S_{k}} E_{i}\right) \triangle J_{k}
$$

is a finite set and that $x_{i} \in J_{k-1} \cup J_{k} \cup J_{k+1}$ when $i \in S_{k}$. It follows that

$$
\begin{aligned}
& \sum_{i \in S_{k}} f\left(x_{i}\right) \mu\left(E_{i}\right) \geq \sum_{i \in S_{k}} m^{\&}\left(f, J_{k}\right) \mu\left(E_{i}\right)=m^{\&}\left(f, J_{k}\right) \ell\left(J_{k}\right) ; \\
& \sum_{i \in S_{k}} f\left(x_{i}\right) \mu\left(E_{i}\right) \leq \sum_{i \in S_{k}} M^{\&}\left(f, J_{k}\right) \mu\left(E_{i}\right)=M^{\&}\left(f, J_{k}\right) \ell\left(J_{k}\right) .
\end{aligned}
$$

Summing these inequalities for $1 \leq k \leq q$ yields

$$
\sum_{k=1}^{q} m^{\&}\left(f, J_{k}\right) \ell\left(J_{k}\right) \leq \sum_{i=1}^{n} f\left(x_{i}\right) \mu\left(E_{i}\right) \leq \sum_{k=1}^{q} M^{\&}\left(f, J_{k}\right) \ell\left(J_{k}\right) .
$$


Using both parts of the conclusion of Lemma 1, we find that

$$
\left|\sum_{i=1}^{n} f\left(x_{i}\right) \mu\left(E_{i}\right)-\int_{a}^{b} f\right|<\epsilon .
$$

As the converse is trivial, the proof is complete. 
The proof for the McShane integral is essentially a consequence of one of Littlewood's three principles - namely, a measurable set is almost a figure (see Royden [3]). We first prove the following lemma.

Lemma 4. Let $\delta$ be a positive function defined on $[a, b]$, let $\left\{x_{i}: 1 \leq i \leq n\right\}$ be a finite set of points in $[a, b]$, let $\left\{H_{i}: 1 \leq i \leq n\right\}$ be a finite collection of disjoint closed sets in $[a, b]$ such that $H_{i} \subseteq\left(x_{i}-\delta\left(x_{i}\right), x_{i}+\delta\left(x_{i}\right)\right)$ for each $i$, and let $\beta>0$. Then there exists a finite collection $\left\{\left(x_{i}, V_{i}\right): 1 \leq i \leq n\right\}$ of non-overlapping $\delta$-fine tagged figures such that $H_{i} \subseteq V_{i}$ and $\mu\left(V_{i} \backslash H_{i}\right)<\beta / n$ for each $i$.

Proof. Since the sets $H_{i}$ are disjoint and compact, there exists a positive number $\alpha$ such that

$$
\inf \left\{|x-y|: x \in H_{i}, y \in H_{j}\right\}>\alpha \text { whenever } i \neq j .
$$

For each index $i$, choose an open set $O_{i}$ such that $x_{i} \in O_{i}$ and

$$
H_{i} \subseteq O_{i} \subseteq\left(x_{i}-\delta\left(x_{i}\right), x_{i}+\delta\left(x_{i}\right)\right) \quad \text { and } \quad \mu\left(O_{i} \backslash H_{i}\right)<\beta / n .
$$

Now fix $i$ and define a positive function $\delta_{i}$ on $[a, b]$ as follows:

if $x \in[a, b] \backslash H_{i}$, then choose $\delta_{i}(x)>0$ so that $\left(x-\delta_{i}(x), x+\delta_{i}(x)\right) \cap H_{i}=\emptyset$;

if $x \in H_{i}$, then choose $0<\delta_{i}(x)<\alpha / 4$ so that $\left(x-\delta_{i}(x), x+\delta_{i}(x)\right) \subseteq O_{i}$. Let $\left\{\left(c_{k}, I_{k}\right): 1 \leq k \leq q\right\}$ be a $\delta_{i}$-fine tagged (interval) partition of $[a, b]$ and let $V_{i}$ be the figure consisting of all the closed intervals $I_{k}$ for which $H_{i} \cap I_{k} \neq \emptyset$ along with the degenerate interval $\left[x_{i}, x_{i}\right]$. By the definition of $\delta_{i}$, we find that

$$
\begin{aligned}
& H_{i} \subseteq V_{i} \subseteq O_{i}, \quad x_{i} \in V_{i} \subseteq\left(x_{i}-\delta\left(x_{i}\right), x_{i}+\delta\left(x_{i}\right)\right), \quad \text { and } \\
& \mu\left(V_{i} \backslash H_{i}\right) \leq \mu\left(O_{i} \backslash H_{i}\right)<\beta / n .
\end{aligned}
$$

Finally, choosing $\delta_{i}(x)<\alpha / 4$ when $x \in H_{i}$ guarantees that the figures $V_{i}$ and $V_{j}$ are non-overlapping when $i$ and $j$ are distinct. This completes the proof.

A familiar result, Henstock's Lemma, makes an appearance in the proof of the next theorem. Anyone with any experience in this field is aware of this lemma. In informal language, it states that once a function $\delta$ has been chosen that "works" for $\delta$-fine tagged figure partitions, the same function "works" for any collection of non-overlapping $\delta$-fine tagged figures. The use of Henstock's Lemma in the following proof should make this statement clear. A proof of this lemma can be found in [1]. 
Theorem 5. A function $f:[a, b] \rightarrow R$ is McShane integrable on $[a, b]$ if and only if there is a number $L$ with the following property: for each $\epsilon>0$ there exists a positive function $\delta$ defined on $[a, b]$ such that

$$
\left|\sum_{i=1}^{n} f\left(x_{i}\right) \mu\left(E_{i}\right)-L\right|<\epsilon
$$

whenever $\left\{\left(x_{i}, E_{i}\right): 1 \leq i \leq n\right\}$ is a $\delta$-fine tagged measurable partition of $[a, b]$.

Proof. Suppose that $f$ is McShane integrable on $[a, b]$ and let $\epsilon>0$. Since $f$ is McShane integrable on $[a, b]$, there exists a positive function $\delta$ defined on $[a, b]$ such that

$$
\left|\sum_{k=1}^{q} f\left(x_{k}\right) \ell\left(V_{k}\right)-\int_{a}^{b} f\right|<\epsilon
$$

whenever $\left\{\left(x_{k}, V_{k}\right): 1 \leq k \leq q\right\}$ is a $\delta$-fine tagged figure partition of $[a, b]$, where $\int_{a}^{b} f$ is the McShane integral of $f$ on $[a, b]$. Since the indefinite McShane integral of $f$ is absolutely continuous on $[a, b]$, there exists $0<\eta<\epsilon$ such that $\left|\int_{V} f\right|<\epsilon$ whenever $V$ is a figure in $[a, b]$ that satisfies $\ell(V)<\eta$. Suppose that $\left\{\left(x_{i}, E_{i}\right): 1 \leq i \leq n\right\}$ is a $\delta$-fine tagged measurable partition of $[a, b]$. Let $M=1+\max \left\{\left|f\left(x_{i}\right)\right|: 1 \leq i \leq n\right\}$, let $E$ be the set of all $x$ in $[a, b]$ such that $x \in E_{i} \cap E_{j}$ for some pair $i, j$ of distinct indices, and note that $E$ is a finite set. For each index $i$, there exists a closed set $H_{i} \subseteq E_{i} \backslash E$ such that $\mu\left(E_{i} \backslash H_{i}\right)<\eta / 2 n M$. Since $\left\{H_{i}: 1 \leq i \leq n\right\}$ is a finite collection of disjoint closed sets in $[a, b]$, the previous lemma states that there exists a finite collection $\left\{\left(x_{i}, V_{i}\right): 1 \leq i \leq n\right\}$ of non-overlapping $\delta$-fine tagged figures such that $H_{i} \subseteq V_{i}$ and $\mu\left(V_{i} \backslash H_{i}\right)<\eta / 2 n M$ for each $i$. It should be clear that there exists a figure $V$ such that $\left\{V_{i}: 1 \leq i \leq n\right\} \cup V$ is a figure partition of $[a, b]$. Since

$$
E_{i} \triangle V_{i}=\left(E_{i} \backslash V_{i}\right) \cup\left(V_{i} \backslash E_{i}\right) \subseteq\left(E_{i} \backslash H_{i}\right) \cup\left(V_{i} \backslash H_{i}\right),
$$

it follows that $\mu\left(E_{i} \triangle V_{i}\right)<\eta / n M$ and

$$
\mu(V)=\mu\left(\bigcup_{i=1}^{n} E_{i} \backslash \bigcup_{i=1}^{n} V_{i}\right) \leq \sum_{i=1}^{n} \mu\left(E_{i} \backslash V_{i}\right)<\frac{\eta}{M} \leq \eta .
$$

Using all of this information and Henstock's Lemma, we obtain

$$
\begin{aligned}
& \left|\sum_{i=1}^{n} f\left(x_{i}\right) \mu\left(E_{i}\right)-\int_{a}^{b} f\right| \\
& \quad=\left|\sum_{i=1}^{n} f\left(x_{i}\right) \mu\left(E_{i}\right)-\sum_{i=1}^{n} f\left(x_{i}\right) \mu\left(V_{i}\right)+\sum_{i=1}^{n} f\left(x_{i}\right) \ell\left(V_{i}\right)-\sum_{i=1}^{n} \int_{V_{i}} f-\int_{V} f\right|
\end{aligned}
$$




$$
\begin{aligned}
& \leq \sum_{i=1}^{n}\left|f\left(x_{i}\right)\right|\left|\mu\left(E_{i}\right)-\mu\left(V_{i}\right)\right|+\left|\sum_{i=1}^{n} f\left(x_{i}\right) \ell\left(V_{i}\right)-\sum_{i=1}^{n} \int_{V_{i}} f\right|+\left|\int_{V} f\right| \\
& \leq M \sum_{i=1}^{n} \mu\left(E_{i} \triangle V_{i}\right)+\epsilon+\epsilon<\eta+\epsilon+\epsilon<3 \epsilon .
\end{aligned}
$$

This completes the proof.

The final comments in this paper concern the use of the Henstock integral to define measure. Once the Henstock integral has been defined without any mention of measure theory and its properties established, one can say that a set $E \subseteq[a, b]$ is integrable if $\chi_{E}$ (the characteristic function of $E$ ) is Henstock integrable on $[a, b]$. It is then possible to use the properties of the Henstock integral to show that the collection of integrable sets possesses the same properties as the collection of measurable sets. Proponents of this definition argue that the simplicity of the Henstock integral makes this a gentler introduction to measure theory. Although I am not in favor of introducing measurable sets in this way (see [2]), the following connection between the Henstock integral and Lebesgue outer measure is of interest in this construction of a measure.

Given a set $E \subseteq[a, b]$ and a positive function $\delta$ defined on $[a, b]$, let

$$
M_{\delta}(E)=\sup \left\{\sum_{k=1}^{q} \chi_{E}\left(x_{k}\right) \ell\left(I_{k}\right)\right\}
$$

where the supremum is taken over all $\delta$-fine tagged partitions $\left\{\left(x_{k}, I_{k}\right): 1 \leq\right.$ $k \leq q\}$ of $[a, b]$, then define

$$
h^{*}(E)=\inf \left\{M_{\delta}(E): \delta \text { is a positive function defined on }[a, b]\right\} .
$$

Lebesgue outer measure will be defined in the usual way as

$$
\begin{aligned}
\mu^{*}(E)=\inf & \left\{\sum_{k=1}^{\infty} \ell\left(I_{k}\right):\left\{I_{k}\right\}\right. \text { is a sequence of open intervals such that } \\
& \left.E \subseteq \bigcup_{k=1}^{\infty} I_{k}\right\} .
\end{aligned}
$$

We will prove that $h^{*}(E)=\mu^{*}(E)$ for every set $E \subseteq[a, b]$. The following lemma, which is due to R. G. Bartle, will be useful in the proof.

Lemma 6. Let $E$ be any set of real numbers. If $\delta$ is a positive function defined on $R$, then there exists a countable collection $\left\{\left(x_{k}, J_{k}\right)\right\}$ of non-overlapping $\delta$-fine tagged intervals such that $x_{k} \in E$ for each $k$ and $E \subseteq \bigcup_{k=1}^{\infty} J_{k}$. 
Proof. It is sufficient to prove the lemma for the case $E \subseteq[0,1]$. Let $\left\{I_{n}\right\}$ be the following sequence of dyadic intervals:

$$
\left[0, \frac{1}{2}\right],\left[\frac{1}{2}, 1\right],\left[0, \frac{1}{4}\right],\left[\frac{1}{4}, \frac{1}{2}\right],\left[\frac{1}{2}, \frac{3}{4}\right],\left[\frac{3}{4}, 1\right],\left[0, \frac{1}{8}\right],\left[\frac{1}{8}, \frac{1}{4}\right], \cdots .
$$

For each $x \in E$, there exists a least positive integer $n_{x}$ such that $x \in I_{n_{x}} \subseteq$ $(x-\delta(x), x+\delta(x))$. The collection $\left\{I_{n_{x}}: x \in E\right\}$ is countable and $E \subseteq$ $\bigcup_{x \in E} I_{n_{x}}$. By removing those intervals in the collection that are contained in other intervals in the collection, we obtain a countable collection $\left\{J_{k}\right\}$ of nonoverlapping closed intervals such that $E \subseteq \bigcup_{k=1}^{\infty} J_{k}$. For each $k$, there exists a point $x_{k} \in E \cap J_{k}$ such that $\left(x_{k}, J_{k}\right)$ is a $\delta$-fine tagged interval. This completes the proof.

Theorem 7. If $E \subseteq[a, b]$, then $h^{*}(E)=\mu^{*}(E)$.

Proof. Let $\left\{I_{k}\right\}$ be a sequence of open intervals such that $E \subseteq \bigcup_{k=1}^{\infty} I_{k}$ and let $O=\bigcup_{k=1}^{\infty} I_{k}$. Define a positive function $\delta$ on $[a, b]$ as follows:

if $x \in E$, then choose $\delta(x)>0$ so that $(x-\delta(x), x+\delta(x)) \subseteq O$;

if $x \in[a, b] \backslash E$, then let $\delta(x)=b-a$.

Let $\left\{\left(x_{i}, K_{i}\right): 1 \leq i \leq q\right\}$ be any $\delta$-fine tagged partition of $[a, b]$ and note that $x_{i} \in E$ implies $K_{i} \subseteq O$. Hence,

$$
\sum_{i=1}^{q} \chi_{E}\left(x_{i}\right) \ell\left(K_{i}\right) \leq \mu(O) \leq \sum_{k=1}^{\infty} \ell\left(I_{k}\right) .
$$

It follows that $h^{*}(E) \leq M_{\delta}(E) \leq \sum_{k=1}^{\infty} \ell\left(I_{k}\right)$. Since the sequence $\left\{I_{k}\right\}$ is arbitrary, we find that $h^{*}(E) \leq \mu^{*}(E)$.

Now let $\delta$ be a positive function defined on $[a, b]$. By Lemma 6 , there exists a countable collection $\left\{\left(x_{k}, J_{k}\right)\right\}$ of non-overlapping $\delta$-fine tagged intervals such that $x_{k} \in E$ for each $k$ and $E \subseteq \bigcup_{k=1}^{\infty} J_{k}$. Without loss of generality, we may assume that $J_{k} \subseteq[a, b]$ for each $k$. Let $\epsilon>0$. By making each of the closed intervals $J_{k}$ a little bigger, we can obtain a sequence $\left\{I_{k}\right\}$ of open intervals such that

$$
E \subseteq \bigcup_{k=1}^{\infty} I_{k} \text { and } \sum_{k=1}^{\infty} \ell\left(I_{k}\right)<\sum_{k=1}^{\infty} \ell\left(J_{k}\right)+\epsilon
$$


Since the $J_{k}$ 's are non-overlapping, we can pick a positive integer $n$ such that $\sum_{k=n+1}^{\infty} \ell\left(J_{k}\right)<\epsilon$, then choose a $\delta$-fine tagged partition $\left\{\left(c_{i}, K_{i}\right): 1 \leq i \leq q\right\}$ of $[a, b]$ that contains the collection $\left\{\left(x_{k}, J_{k}\right): 1 \leq k \leq n\right\}$. Since $x_{k} \in E$ for each $k$,

$$
\begin{aligned}
\sum_{k=1}^{\infty} \ell\left(I_{k}\right) & <\sum_{k=1}^{\infty} \ell\left(J_{k}\right)+\epsilon<\sum_{k=1}^{n} \ell\left(J_{k}\right)+2 \epsilon=\sum_{k=1}^{n} \chi_{E}\left(x_{k}\right) \ell\left(J_{k}\right)+2 \epsilon \\
& \leq \sum_{i=1}^{q} \chi_{E}\left(c_{i}\right) \ell\left(K_{i}\right)+2 \epsilon \leq M_{\delta}(E)+2 \epsilon .
\end{aligned}
$$

It follows that $\mu^{*}(E) \leq M_{\delta}(E)+2 \epsilon$. Since $\epsilon>0$ was arbitrary, $\mu^{*}(E) \leq$ $M_{\delta}(E)$. Since this is true for every positive function $\delta$ defined on $[a, b]$, we obtain $\mu^{*}(E) \leq h^{*}(E)$. This completes the proof.

It follows that the collection of integrable sets is identical to the collection of measurable sets. A discussion of the properties of this measure can either follow the standard line of argument or appeal to the properties of the Henstock integral.

\section{References}

[1] R. A. Gordon, The integrals of Lebesgue, Denjoy, Perron, and Henstock, Graduate Studies in Mathematics, Vol. 4, American Mathematical Society, Providence, RI, 1994.

[2] R. A. Gordon, Is nonabsolute integration worth doing?, Real Analysis Exchange, 22 (1996-97), 23-33.

[3] H. L. Royden, Real analysis, 3rd ed., Macmillan, New York, 1988. 